\title{
Isolation and Molecular Characterization of a Model Antagonistic Pseudomonas aeruginosa Divulging In Vitro Plant Growth Promoting Characteristics
}

\author{
Bushra Uzair (D), ${ }^{1}$ Rehana Kausar ${ }^{1},{ }^{2}$ Syeda Asma Bano, ${ }^{3}$ Sammer Fatima, ${ }^{4}$ Malik Badshah, ${ }^{5}$ \\ Ume Habiba, ${ }^{6}$ and Fehmida Fasim ${ }^{7}$ \\ ${ }^{1}$ Department of Bioinformatics and Biotechnology, International Islamic University, Islamabad Capital Territory, \\ Islamabad 44000, Pakistan \\ ${ }^{2}$ Department of Botany, University of Azad Jammu and Kashmir, Muzaffarabad 13100, Pakistan \\ ${ }^{3}$ Department of Microbiology, University of Haripur, Haripur, Pakistan \\ ${ }^{4}$ Department of Botany, University of Gujrat, Hafiz Hayat Campus, Gujrat, Pakistan \\ ${ }^{5}$ Department of Microbiology, Quaid-i-Azam University, Islamabad 45320, Pakistan \\ ${ }^{6}$ Department of Forestry and Wild Life Management, University of Haripur, Haripur, Pakistan \\ ${ }^{7}$ Discipline of Biomedical Science, Sydney Medical School, The University of Sydney, Sydney, NSW, Australia
}

Correspondence should be addressed to Bushra Uzair; bushra.uzair@iiu.edu.pk

Received 25 August 2017; Accepted 27 November 2017; Published 15 January 2018

Academic Editor: Rida Batool

Copyright (C) 2018 Bushra Uzair et al. This is an open access article distributed under the Creative Commons Attribution License, which permits unrestricted use, distribution, and reproduction in any medium, provided the original work is properly cited.

\begin{abstract}
The use of microbial technologies in agriculture is currently expanding quite rapidly with the identification of new bacterial strains, which are more effective in promoting plant growth. In the present study 18 strains of Pseudomonas were isolated from soil sample of Balochistan coastline. Among isolated Pseudomonas strains four designated as SP19, SP22, PS24, and SP25 exhibited biocontrol activities against phytopathogenic fungi, that is, Rhizopus microsporus, Fusarium oxysporum, Aspergillus niger, Alternaria alternata, and Penicillium digitatum; PS24 identified as Pseudomonas aeruginosa by 16srRNA gene bank accession number EU081518 was selected on the basis of its antifungal activity to explore its potential as plant growth promotion. PS24 showed multiple plant growth promoting attributes such as phosphate solubilization activity, indole acetic acid (IAA), siderophore, and HCN production. In order to determine the basis for antifungal properties, antibiotics were extracted from King B broth of PS24 and analyzed by TLC. Pyrrolnitrin antibiotic was detected in the culture of strain PS24. PS24 exhibited antifungal activities found to be positive for hydrogen cyanide synthase Hcn BC gene. Sequencing of gene of Hcn BC gene of strain PS24 revealed 99\% homology with the Pseudomonas aeruginosa strain PA01. The sequence of PS24 had been submitted in gene bank accession number KR605499. Ps. aeruginosa PS24 with its multifunctional biocontrol possessions can be used to bioprotect the crop plants from phytopathogens.
\end{abstract}

\section{Introduction}

Pseudomonas are ubiquitous bacteria which are of industrial significance. This is because they exhibit various traits in a wide variety of environments. Pseudomonas influences plant and animal pathogens. Use of Pseudomonas spp. includes biocontrol and as a plant growth promoter and an efficient bioremediation strain and also for production of array of antimicrobial compounds [1, 2]. Evidence has highlighted many metabolites produced by Pseudomonas strains such as antibiotics, enzymes, and volatiles and their key role in controlling an array of fungi causing infections in plants has been used for biofertilizer production and formulation $[3,4]$. Toxins liberated from plant pathogenic fungi cause budding infectious diseases leading to epidemics and concerns for food safety [5]. Furthermore, plant pathogenic fungi threaten plant and have been long recognized as a prevalent problem. There are various epidemics reported because of plant disease caused by microorganisms and have threatened food security in history of mankind [6]. Due to the impact of 
pathogenic fungi on human life, significant research efforts have been directed towards combating fungal infections by identifying novel targets for development of antifungal agents [7, 8]. Many Antimicrobial compounds, surfactants, and plant growth promoters have been reported by potential plant growth promoting strains colonizing plant roots and bacterial secretions regulate the interaction between plants and PGPR leading to distinctive plant growth promotion special effects $[9,10]$. Genetically improved strains with greater plant protection traits were also designed [11]. Consortium of $P$. fluorescens with other plant growth promoting beneficial bacteria strains showed promising plant growth promoting traits [12].

In this study, a strain of Pseudomonas aeruginosa PS24 which is believed to be a plant growth promoting microorganism was obtained from soil samples of Balochistan coastline (Pakistan). Strains were evaluated to see if they had characteristics important for sustainable agriculture. These characteristics include biological control protection, phosphorous solubilization, protease production, $\mathrm{HCN}$, plant growth promoting hormone (indole acetic acid), and siderophore production. HCN gene cluster from Pseudomonas aeruginosa PS24 was also amplified and sequenced.

\section{Material and Methods}

2.1. Sample Collection and Isolation of Pseudomonas Strains. Coastline soils samples were collected from Balochistan coastline of Pakistan. Physicochemical analysis of samples based on soil texture, $\mathrm{pH}$, and temperature was examined. The soil texture was slit clay type, $\mathrm{pH}$ in the range of 7.5 to 8.8 , and temperature was 25 to $32^{\circ} \mathrm{C}$. Microbial strains were isolated by the serial dilution method. One gram of dried soil was weighed and added to $9 \mathrm{ml}$ of double distilled water ( $\mathrm{dd}_{2} \mathrm{O}$ ) in a sterile test tube and shaken well using vortex mixer; this stock solution was then diluted serially up to the dilution of $10^{-5}$ and $0.1 \mathrm{~mL}$ of diluted sample was inoculated on surface of selective King's B agar and incubated at $30^{\circ} \mathrm{C}$ for 2 days [13]. The purified colonies were preserved using standard preservation methods.

2.2. Identification of the Selected Bacteria. After isolation of strains on selective King B medium the strains were identified and characterized by morphological, cultural, and biochemical tests using Bergey's manual as a reference [14]. Identification was also confirmed by $16 \mathrm{~S}$ rRNA gene sequencing by using GAGTTTGATCCTGGCTCAG and AGAAAGGAGGTATCCAGCC forward and reverse primer sequence, respectively.

2.3. Production of Antifungal Compounds by Pseudomonas Strains. In this study all together 18 strains of Pseudomonas were examined for exploring antifungal activity of strains by dual culture assay using method described by [15].

2.4. Antimicrobial Metabolites Produced by PS24. Ability of PS24 to produce siderophore was examined using method described by Alexander and Zuberer 1991 [16]. Using Bakker and Schippers 1987 [17] method HCN production was determined.

2.5. Determination of Phosphorous Solubilization and Protease Production Activity. The phosphate solubilization ability of Pseudomonas PS24 strain was determined by using, Mehta and Nautiyal, protocol [18]. A single colony was streaked onto the tris minimal medium supplemented with $0.8 \mathrm{mM}$ calcium triphosphate and $0.5 \mathrm{mM}$ glucose. After 3 days of incubation at $30^{\circ} \mathrm{C}$, a clear zone around the colony was observed and measured. Protease production was done on skim milk agar plates containing $2.5 \%$ skim milk.

2.6. Estimation of Inorganic Phosphate by HPLC. At different intervals of time release of free inorganic phosphate in tris minimal liquid medium with the addition of $5 \mathrm{mM}$ calcium triphosphate by PS24 was determined by HPLC. Aseptically drawn $10 \mathrm{ml}$ aliquot from each flask was taken and centrifuged at $5000 \mathrm{~g}$ for $15 \mathrm{~min}$. Pellet was discarded and supernatant was assessed for phosphate content using column (AS11-HC/AG11-HC). The phosphate content was quantitatively analyzed by comparing the retention times and peak areas of chromatograms with standards.

2.7. Screening for Indole Acetic Acid (IAA) Production. Method of Ehmann, 1977, was used to screen IAA production using Salkowski's reagent [19]. The strain Pseudomonas PS24 was grown in tryptone soy broth and incubated for 4 days. The broth was centrifuged and supernatant was mixed with $2 \mathrm{ml}$ of Salkowski's reagent and kept in the dark. Development of pink color is indicative of indole acetic acid (IAA) production [19].

2.8. Analysis of Antibacterial Compounds from PS24. From PS24 grown on King B broth antimicrobial compounds were extracted using combination of extracting solvents, ethyl acetate, and $80 \%$ acetone in equal volume. TLC of the prepared crude extract was performed with precoated silica gel G25-UV254 plates and was observed under UV light $(254 \mathrm{~nm})$. The distinct separated fractions were recovered in DMSO and tested for antifungal activity.

2.9. Exploring Antifungal Compound Producing Genes of Pseudomonas and Its Sequencing. Selected strain producing highest zone of inhibition was subjected to the detection of Hcn BC gene using Primer Aca and Acb of Ramette et al., 2003 [20]. The sequence of primers for PCR amplification used was Aca ACTGCCAGGGGCGGATGTGC and Acb ACGATGTGCTCGGCGTAC. Sequencing of Hydrogen Cyanide Synthase Gene of Pseudomonas was also done. Sequencing was run using ABI3130 Genetic Analyzer.

\section{Results}

3.1. Isolation and Identification of Antagonistic Bacteria Strains. All 18 strains of Pseudomonas recovered from soil samples of Balochistan coastline were Gram-negative and positive for citrate, oxidase, catalase-, indole, and produced 


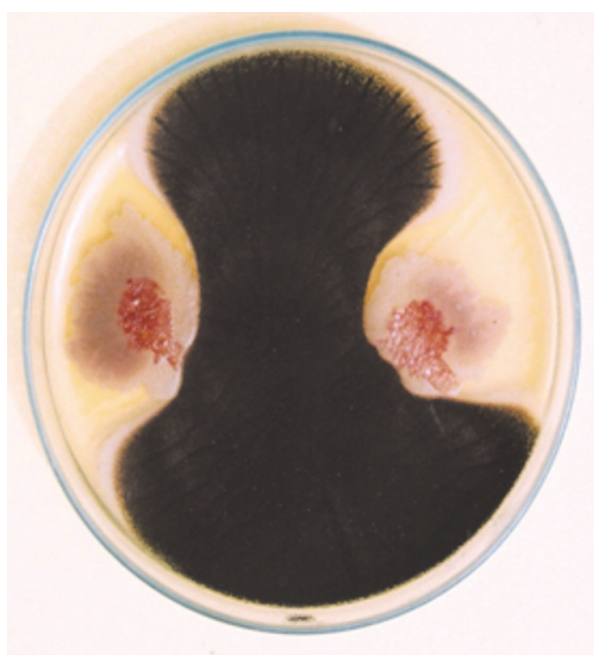

(a)

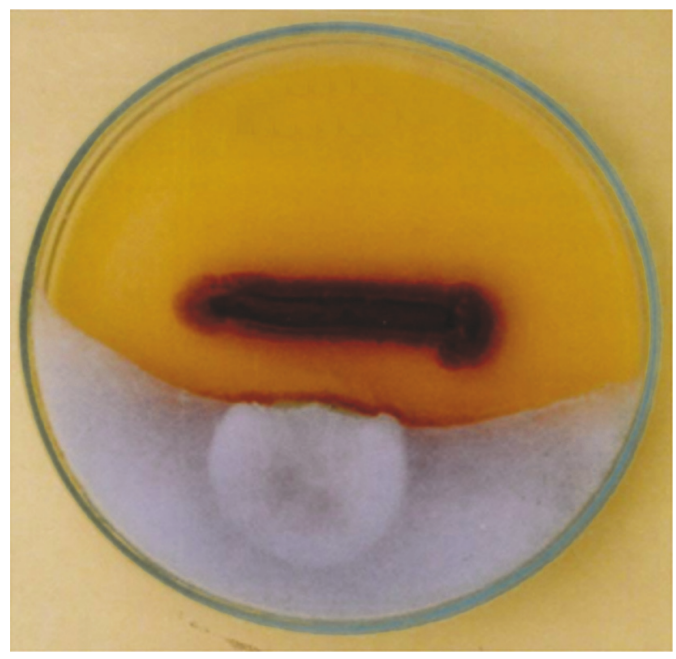

(b)

FIgURE 1: Screening of Pseudomonas strains PS24 for antifungal activity against plant pathogens, (a) inhibition of Alternaria alternata; (b) inhibition of Rhizopus microsporus.

TABLE 1: Biochemical characteristics and estimation of various biological traits of PS24.

\begin{tabular}{lc}
\hline Test & Trait \\
\hline Auxin production & Positive \\
Siderophore & Positive \\
Phosphorous solubilization & Positive \\
HCN production & Positive \\
\hline & \\
\hline Rhizopus microspores & Antifungal activity \\
Fusarium oxysporum, & ++++ \\
Aspergillus niger & +++ \\
Alternaria alternata & ++ \\
Penicillium digitatum & ++ \\
&
\end{tabular}

siderophores and inert reaction was observed on triple sugar iron agar slants. The strain was identified as Pseudomonas aeruginosa accession number EU081518. These strains were screened for antifungal activity to inhibit growth of fungal strains. Four strains showed effectual antagonistic activity towards Rhizopus microsporus, Fusarium oxysporum, Aspergillus niger, Alternaria alternata, and Penicillium digitatum. Among them, the bacterial strain PS24 was the leading one as antagonist strain against fungal pathogens. Therefore PS24 strain was used for further experiments in search of potential strain that can be employed as biofertilizer strain. The colony of strain of PS24 was orangish yellow pigmented, shiny, and exhibiting an irregular margin. Identification characteristics are presented in (Table 1).

3.2. Assessment of Antifungal Activity. The cross-streak method and dual culture assay of the culture/culture filtrate of strain PS24 showed antifungal nature by both tested methods for evaluation of antifungal potential. Antifungal activity was observed with Fusarium oxysporum, Aspergillus niger, Alternaria alternata, and Penicillium digitatum (Figure 1; Table 2).

3.3. Screening for Plant Growth Promoting Characteristics of PS24. Both the phosphate solubilizing strains showed manifestation of a reddish-brown zone around the colony on CAS agar plates which confirmed the siderophore production (Figure 2). This strain also displayed production of indole acetic acid (Figure 2).

3.4. Estimation of Inorganic Phosphate by HPLC. The presence of soluble phosphate in the medium after different intervals of time ( 0 day, to 20 days) was confirmed by HPLC. Results are shown in (Figure 3). PS24 solubilized calcium triphosphate in liquid medium with connected release of free phosphate in the medium HPLC analysis showed that the quantity of phosphate in the medium demonstrated a steady boost and attained the highest level after 10 days of incubation.

\subsection{Chromatographic Identification of the Antibiotic Produced} by Strain PS24. Antimicrobial compounds extracted out from strain PS24 and reference strain Pf-5 grown in King $B$ were spotted on TLC reverse phase silica gel plates that illustrated a pyrrolnitrin band as produced by reference strain Pf-5 (Figure 4). Separated pyrrolnitrin compound on TLC plate showed antifungal activity (Figure 4).

3.6. Screening for the Antifungal Compound Producing by PCR and Sequencing of Hydrogen Cyanide Synthase Gene. Four potential biocontrol strains showed clear bands on agarose gel for $H c n B C$ gene with known primers Aca and Acb and the band size was approx. $586 \mathrm{bp}$ (Figure 5). Sequencing of Hydrogen Cyanide Synthase Gene showed 99\% homology 
TABLE 2: In vitro antifungal activity of PS24.

\begin{tabular}{lcc}
\hline Test fungi & $\begin{array}{c}\text { Size of zone of inhibition (mm) } \\
\text { Culture filtrate of PS24 }\end{array}$ & $\begin{array}{c}\text { Size of zone of inhibition (mm) } \\
\text { Crude extract of PS24 }\end{array}$ \\
\hline Candida albicans & - & - \\
Candida tropicalis & - & - \\
Rhizopus microsporus & 15.5 & 16.0 \\
Fusarium oxysporum, & 14.8 & 15.0 \\
Aspergillus niger & 16.6 & 18.4 \\
Alternaria alternata & 14.5 & 16.5 \\
\hline
\end{tabular}

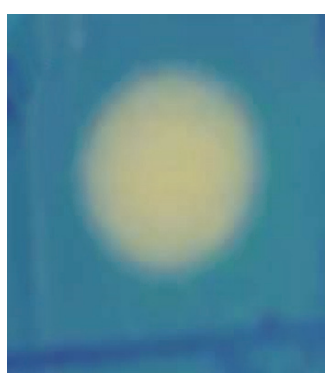

(a)

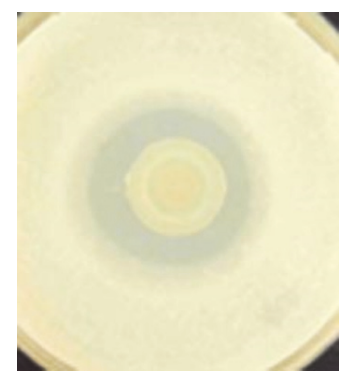

(b)

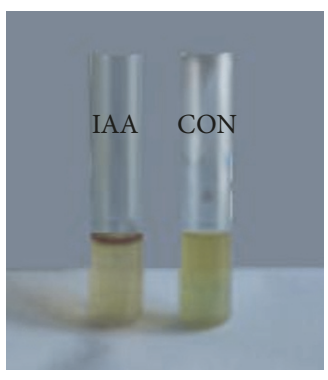

(c)

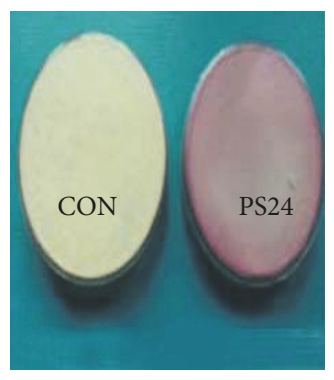

(d)

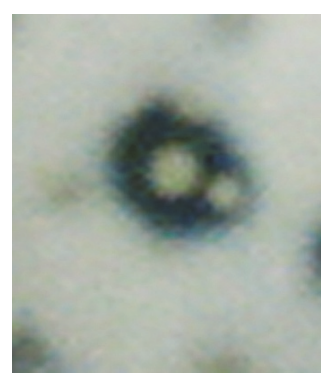

(e)

Figure 2: Plant Growth Promoting Characteristics of strain PS24. (a) Siderophore production. (b) Phosphate solubilization. (c) Indole acetic acid IAA production. (d) HCN production. (e) protease production.

with the Pseudomonas aeruginosa strain PA 01. The sequence of PS24 had been submitted in gene bank and the accession number of this sequence is KR605499.

\section{Discussion}

Pakistan's $1050 \mathrm{~km}$ coastline stretches along the Sindh and Balochistan Province. The greater stretch being in Balochistan of $800 \mathrm{~km}$ and the remainder $250 \mathrm{~km}$ stretch along Sindh, Pakistan, receives less than $250 \mathrm{~mm}$ of rainfall annually which makes the climate semiarid and arid. The coastline is very diverse with regard to marine flora and fauna. Commercially important species can be found intertidal, near the shore and on off shore areas too [21]. Evolution of a variety of highly adapted living organisms is due to the temporal and climatic selection process. These living organisms are a wealthy source of microbial diversity [22]. Unfortunately, very few studies reflect on the diversity of microorganisms found in Balochistan coastline. The present study is based on exploration of potential biofertilizer strains from soil samples of Balochistan coastline of Pakistan. Bacterial plant growth promotion is a complex, well-known and established phenomena. A variety of phenotypic and genotypic characteristics are involved in influencing plant growth in a vast array of agricultural environments as indicated by plant growth promoting bacterial strains [23, 24]. In the present study, Pseudomonas strains were screened for their potential to be used for sustainable agriculture as a possible biocontrol and plant growth promoting agent. Among the isolated strains Pseudomonas strain PS24 identified as Pseudomonas aeruginosa was studied for holding a unique plant growth promoting activity. This was based on its biocontrol property and phosphorous solubilizing activity. This property has been appraised and key information proposes insight into this organism's alternative of biocontrol capabilities. Pseudomonas spp. are distinguished by their production of colorful secondary metabolites called phenazines [25] and strain PS24 produced yellowish orange pigments in King $\mathrm{B}$ agar medium. Antimicrobial compounds were extracted from PS24 grown on King B medium and separated on TLC plates. The distinct separated fractions revealed antifungal potential as shown in Figure 4. In this study, we have obtained efficient detection of pyrrolnitrin on TLC when compared with other known strains. Another significant attribute could be better availability of phosphorous because of P-solubilization by the strain PS24 both on solid and liquid medium as confirmed by plate assay and HPLC analysis in liquid tris minimal medium supplemented with calcium triphosphate. It is well established that enhanced phosphorous nutrition powers general plant growth and root maturity of various economically important plants. Siderophore for iron provision of plants grown under iron scarce conditions and $\mathrm{HCN}$ production is measured valuable from the biocontrol viewpoint. Many studies have shown and have been carried out to highlight the nature and properties of specific microbes which exhibit plant growth promoting properties. Along with chemical fertilizers it is important to seek region-specific strains of microorganisms which can be used in plant growth promotion [26].

Paulsen et al., 2005, [27] showed the complete genome sequence of the Pseudomonas fluorescens Pf-5 (a plant commensal). In this study the presence of $\mathrm{Hcn} \mathrm{BC}$ gene was also explored. Furthermore, sequencing of Hcn BC gene was done. Rijavec and Lapanje, 2016, [28] reported hydrogen 


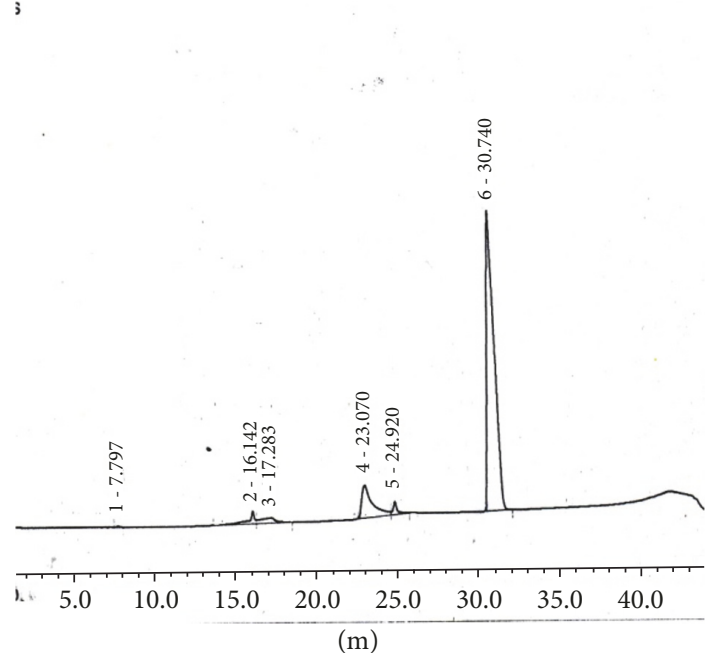

(a)

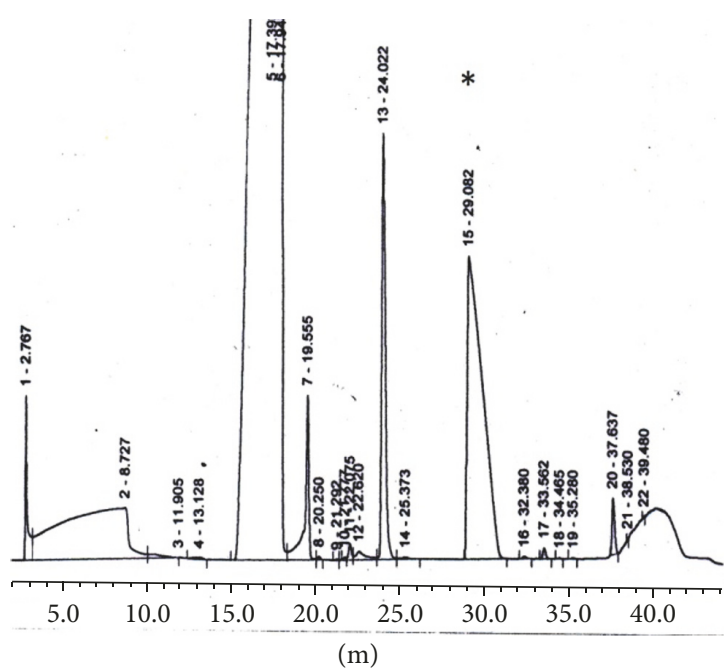

(b)

FIGURE 3: HPLC chromatogram showing free phosphate release. (a) Standard of phosphate ( $5 \mathrm{mM})$, (b) phosphate release by PS24 as a result of solubilization of calcium triphosphate in tris minimal medium supplemented with $5 \mathrm{mM}$ calcium triphosphate. Key. The peak that elutes at $30 \mathrm{~min}$ (indicated with an asterisk) represents phosphate. (a) Standard of phosphate. (b) Phosphate release at 10th day of incubation.

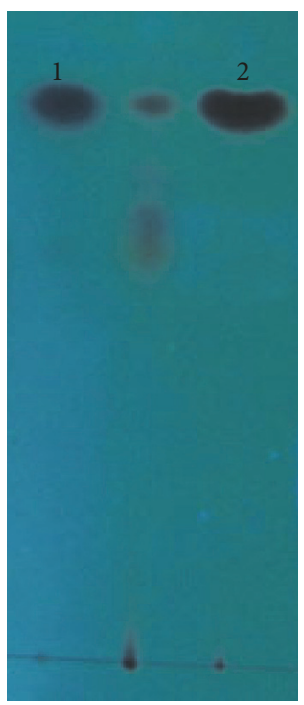

(a)

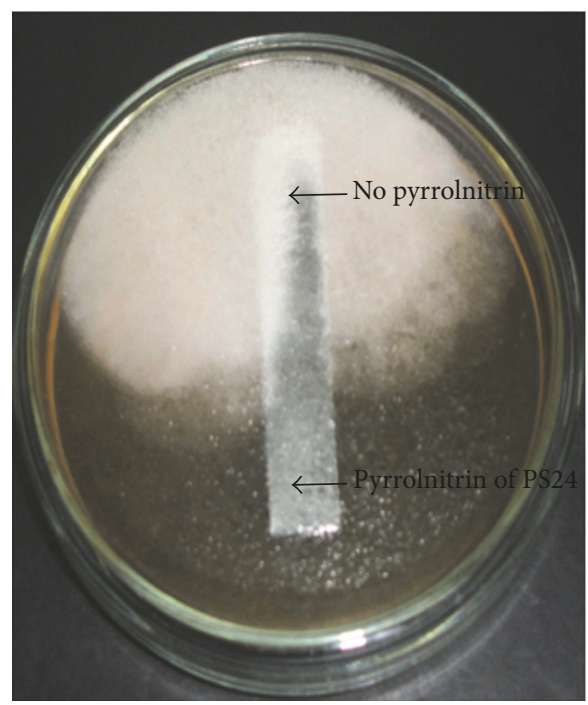

(b)

FIGURE 4: (a) TLC showing compounds produced by Pseudomonas strains Lane 1: Pf-5 reference strain; Lane 2: PS24. (b) Antifungal activity of pyrrolnitrin of PS24 separated on TLC.

cyanide composite is not vital or necessary for progress, energy stowing, or primary metabolism in $P$. aeruginosa but it can offer some environmental advantages to the bacterium by regulating availability of phosphate. De Souza et al., 2003, [29] reported conservation of the response regulator gene gacA in Pseudomonas species. Whole genome sequences are now available for two biocontrol strains belonging to the P. fluorescens lineage (strain SBW25) or a closely related species (strain Pf-5), as well as non-biocontrol P. fluorescens $\mathrm{Pf0}$-1. Comparative genomics [9], gene array-based expression studies [30], and integrated, in situ molecular analyses of microbe-host interactions [4] have started to provide advanced knowledge on plant protection properties and rhizosphere competence of these biocontrol agents. The present study is an attempt to untangle secondary metabolism in Ps. aeruginosa strain PS24 that may have a direct role in its ability to suppress fungal pathogens.

\section{Conclusion}

Antagonistic Pseudomonas aeruginosa PS24 isolated from soil sample of Balochistan coastline of Pakistan have shown promising biocontrol abilities, which are closely linked with the production of antifungal compound Pyrrolnitrin This is 


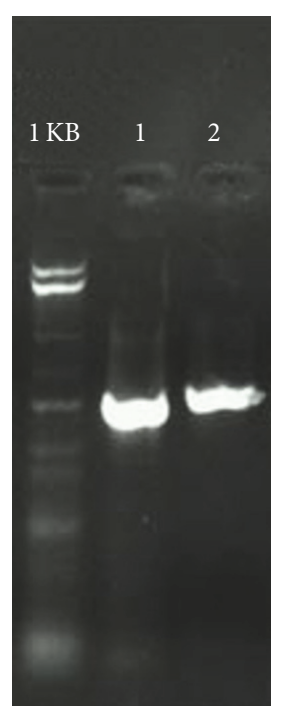

FIgURE 5: Hcn gene Amplification by PCR, strains positive for $\mathrm{Hcn}$ gene showed band size of approx. $586 \mathrm{bp}$. Lane 1 contains PCR product of reference strain Pf5 and lane 2 contains PCR product of PS24.

one of the few reports dealing with isolation and characterization of Pseudomonas aeruginosa strain from soil sample of Balochistan coastline with biocontrol activity against the common soil-borne phytopathogenic fungi in this context; it is proposed that Pseudomonas aeruginosa PS24 can be deployed as an inoculant to attain the desired plant growth promoting action in agricultural environment. Hence it is important to search for region-specific microbial strains which can be used as a potential plant growth promoter to achieve desired product. In this study, we reported Pseudomonas aeruginosa strain with the production of $\mathrm{HCN}$, Pyrrolnitrin, IAA, P-solubilization, and siderophore production as a potential plant growth promoting strain and can be used as bioinoculants in agricultural environments. Thus, the joint developments in environmental biotechnology will support us to comprehend the establishment of Pseudomonas strains as model biocontrol agents, and their right and on time use in rhizosphere and understanding of their behavior and performance in natural soil system for the growth promotion of economically important plants might help us overcome existing tailback curbing their commercial use.

\section{Conflicts of Interest}

The authors declare that they have no conflicts of interest.

\section{References}

[1] D. M. Weller, "Pseudomonas biocontrol agents of soilborne pathogens: Looking back over 30 years," Journal of Phytopathology, vol. 97, no. 2, pp. 250-256, 2007.

[2] A. Upadhyay and S. Srivastava, "Characterization of a new isolate of Pseudomonas fluorescens strain Psd as a potential biocontrol agent," Letters in Applied Microbiology, vol. 47, no. 2, pp. 98-105, 2008.
[3] D. Haas and G. Défago, "Biological control of soil-borne pathogens by fluorescent pseudomonads," Nature Reviews Microbiology, vol. 3, no. 4, pp. 307-319, 2005.

[4] N. Vassilev, M. Vassileva, A. Lopez et al., "Unexploited potential of some biotechnological techniques for biofertilizer production and formulation," Applied Microbiology and Biotechnology, vol. 99, no. 12, pp. 4983-4996, 2015.

[5] C. Matthew, A. Fisher, J. D. Henk et al., "Emerging fungal threats to animal, plant and ecosystem health," Nature, vol. 484, no. 7393, pp. 186-194, 2012.

[6] P. K. Anderson, A. A. Cunningham, N. G. Patel, F. J. Morales, P. R. Epstein, and P. Daszak, "Emerging infectious diseases of plants: Pathogen pollution, climate change and agrotechnology drivers," Trends in Ecology \& Evolution, vol. 19, no. 10, pp. 535544, 2004.

[7] Z. Zhang and L. S. Pierson III, "A Second Quorum-Sensing System Regulates Cell Surface Properties but Not Phenazine Antibiotic Production in Pseudomonas aureofaciens," Applied and Environmental Microbiology, vol. 67, no. 9, pp. 4305-4315, 2001.

[8] A. Ramette, M. Frapolli, G. Défago, and Y. Moënne-Loccoz, "Phylogeny of HCN synthase-encoding honBC genes in biocontrol fluorescent pseudomonads and its relationship with host plant species and HCN synthesis ability," Molecular PlantMicrobe Interactions, vol. 16, no. 6, pp. 525-535, 2003.

[9] D. Zhou, X.-F. Huang, J. M. Chaparro et al., "Root and bacterial secretions regulate the interaction between plants and PGPR leading to distinct plant growth promotion effects," Plant and Soil, vol. 401, no. 1-2, pp. 259-272, 2016.

[10] T. L. G. Carvalho, H. G. F. Ballesteros, F. Thiebaut, P. C. G. Ferreira, and A. S. Hemerly, "Nice to meet you: genetic, epigenetic and metabolic controls of plant perception of beneficial associative and endophytic diazotrophic bacteria in nonleguminous plants," Plant Molecular Biology, vol. 90, no. 6, pp. 561-574, 2016.

[11] G. L. Mark, J. M. Dow, P. D. Kiely et al., "Transcriptome profiling of bacterial responses to root exudates identifies genes involved in microbe-plant interactions," Proceedings of the National Acadamy of Sciences of the United States of America, vol. 102, no. 48, pp. 17454-17459, 2005.

[12] V. Karthikeyan and S. S. Gnanamanickam, "Biological control of Setaria blast (Magnaporthe grisea) with bacterial strains," Crop Protection, vol. 27, no. 2, pp. 263-267, 2008.

[13] E. O. King, M. K. Ward, and D. E. Raney, "Two simple media for the demonstration of pyocyanin and fluorescin," The Journal of Laboratory and Clinical Medicine, vol. 44, no. 2, pp. 301-307, 1954.

[14] J. F. MacFaddin, Biochemical Tests for Identification of Medical Bacteria, Williams and Wilkins, London, UK, 2000.

[15] G. N. Bordoloi, B. Kumari, A. Guha et al., "Isolation and structure elucidation of a new antifungal and antibacterial antibiotic produced by Streptomyces sp. 201," Bioscience, Biotechnology, and Biochemistry, vol. 65, no. 8, pp. 1856-1858, 2001.

[16] D. B. Alexander and D. A. Zuberer, "Use of chrome azurol $S$ reagents to evaluate siderophore production by rhizosphere bacteria," Biology and Fertility of Soils, vol. 12, no. 1, pp. 39-45, 1991.

[17] A. W. Bakker and B. Schippers, "Microbial cyanide production in therhizosphere in relation to potato yield reduction and mediated plant growth stimulation," Soil Biology \& Biochemistry, vol. 19, pp. 451-457, 1987. 
[18] S. Mehta and C. S. Nautiyal, "An efficient method for qualitative screening of phosphate-solubilizing bacteria," Current Microbiology, vol. 43, no. 1, pp. 51-56, 2001.

[19] A. Ehmann, “The van URK-Salkowski reagent - a sensitive and specific chromogenic reagent for silica gel thin-layer chromatographic detection and identification of indole derivatives," Journal of Chromatography A, vol. 132, no. 2, pp. 267-276, 1977.

[20] A. Ramette, Y. Moënne-Loccoz, and G. Défago, "Genetic diversity and biocontrol potential of fluorescent pseudomonads producing phloroglucinols and hydrogen cyanide from Swiss soils naturally suppressive or conducive to Thielaviopsis basicolamediated black root rot of tobacco," FEMS Microbiology Ecology, vol. 55, no. 3, pp. 369-381, 2006.

[21] P. J. A. Siddiqui, S. Farooq, S. Shafique, Z.-U. Burhan, and Z. Farooqi, "Conservation and management of biodiversity in Pakistan through the establishment of marine protected areas," Ocean \& Coastal Management, vol. 51, no. 5, pp. 377-382, 2008.

[22] P. J. A. Siddiqui, S. N. Mansoor, B. Zaib-un-Nisa et al., "Associated fauna and flora of macro-algal puffs inhabiting mangrove stands at Sandspit backwaters, Karachi," Pakistan Journal of Marine Biology, vol. 6, pp. 43-53, 2000.

[23] S. Spaepen, S. Bossuyt, K. Engelen, K. Marchal, and J. Vanderleyden, "Phenotypical and molecular responses of Arabidopsis thaliana roots as a result of inoculation with the auxinproducing bacterium Azospirillum brasilense," New Phytologist, vol. 201, no. 3, pp. 850-861, 2014.

[24] M. A. Trujillo-Roldán, N. A. Valdez-Cruz, C. F. GonzalezMonterrubio et al., "Scale-up from shake flasks to pilot-scale production of the plant growth-promoting bacterium Azospirillum brasilense for preparing a liquid inoculant formulation," Applied Microbiology and Biotechnology, vol. 97, no. 22, pp. 9665-9674, 2013.

[25] O. V. Mavrodi, B. B. M. Gardener, D. V. Mavrodi et al., "Genetic diversity of phlD from 2, 4-diacetylphloroglucinol-producing fluorescent Pseudomonas spp. Phytopathology," Phytopathology, vol. 91, pp. 35-43, 2001.

[26] S. D. Veresoglou and G. Menexes, "Impact of inoculation with Azospirillum spp. on growth properties and seed yield of wheat: A meta-analysis of studies in the ISI Web of Science from 1981 to 2008," Plant and Soil, vol. 337, no. 1, pp. 469-480, 2010.

[27] I. T. Paulsen, C. Press, J. Ravel et al., "Complete genome sequence of the plant commensal Pseudomonas fluorescens Pf5: Insights into the biological control of plant disease," Nature Biotechnology, vol. 23, pp. 873-878, 2005.

[28] T. Rijavec and A. Lapanje, "Hydrogen cyanide in the rhizosphere: Not suppressing plant pathogens, but rather regulating availability of phosphate," Frontiers in Microbiology, vol. 7, pp. 1-14, 2016.

[29] J. T. De Souza, M. Mazzola, and J. M. Raaijmakers, "Conservation of the response regulator gene gacA in Pseudomonas species," Environmental Microbiology, vol. 5, no. 12, pp. 13281340, 2003.

[30] M. Barret, P. Frey-Klett, M. Boutin et al., "The plant pathogenic fungus Gaeumannomyces graminis var. tritici improves bacterial growth and triggers early gene regulations in the biocontrol strain Pseudomonas fluorescens Pf29Arp," New Phytologist, vol. 181, no. 2, pp. 435-447, 2009. 


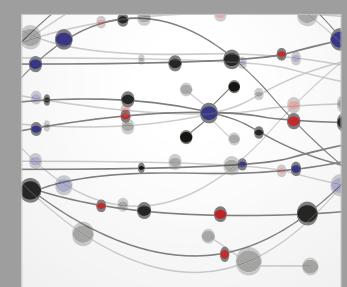

The Scientific World Journal
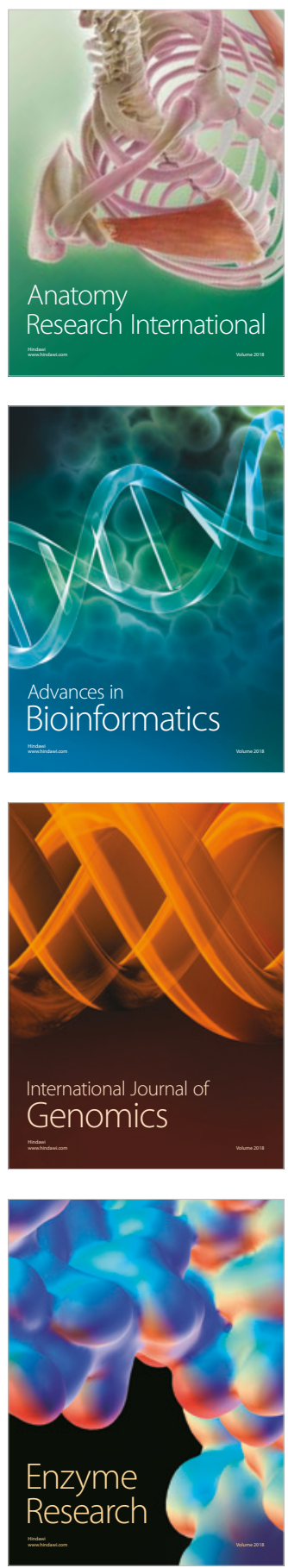
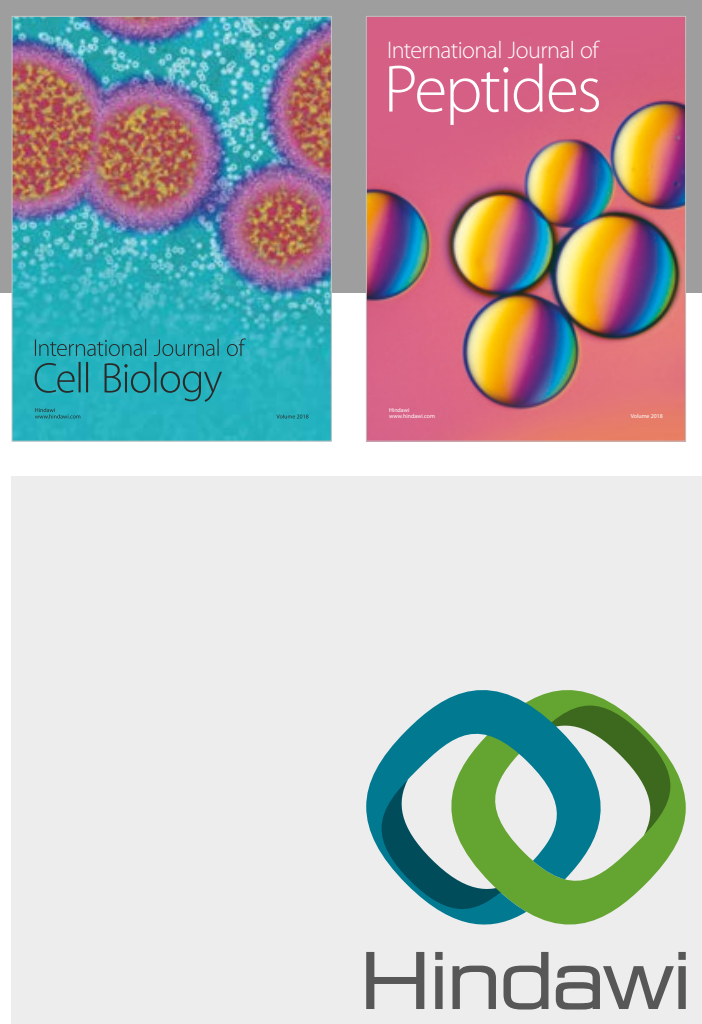

Submit your manuscripts at

www.hindawi.com
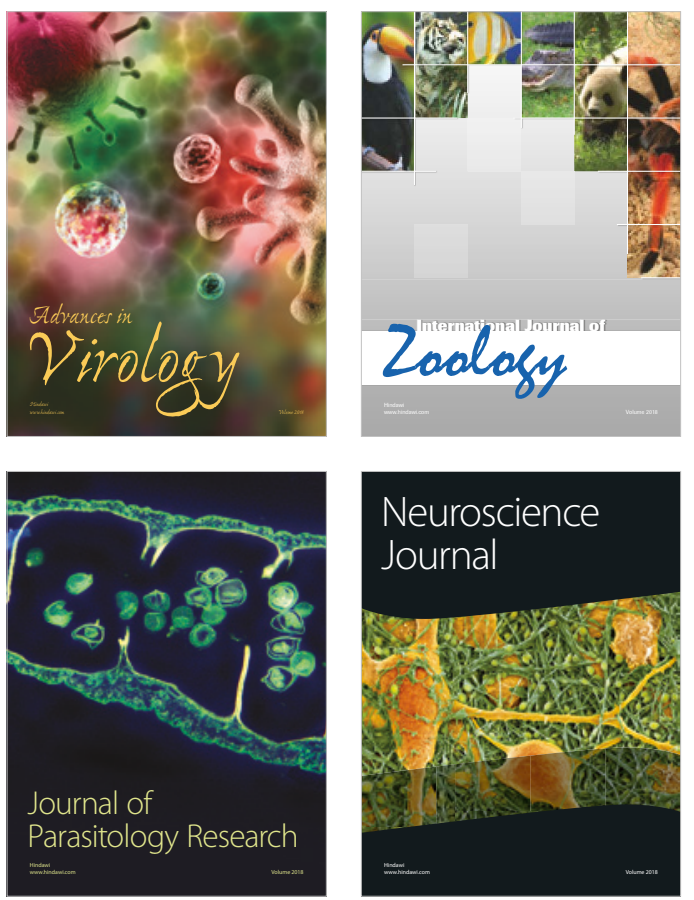
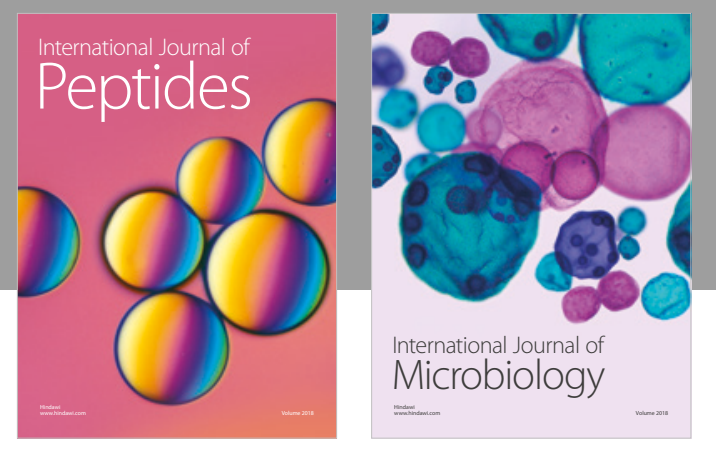

nternational Journal of Microbiology
Journal of
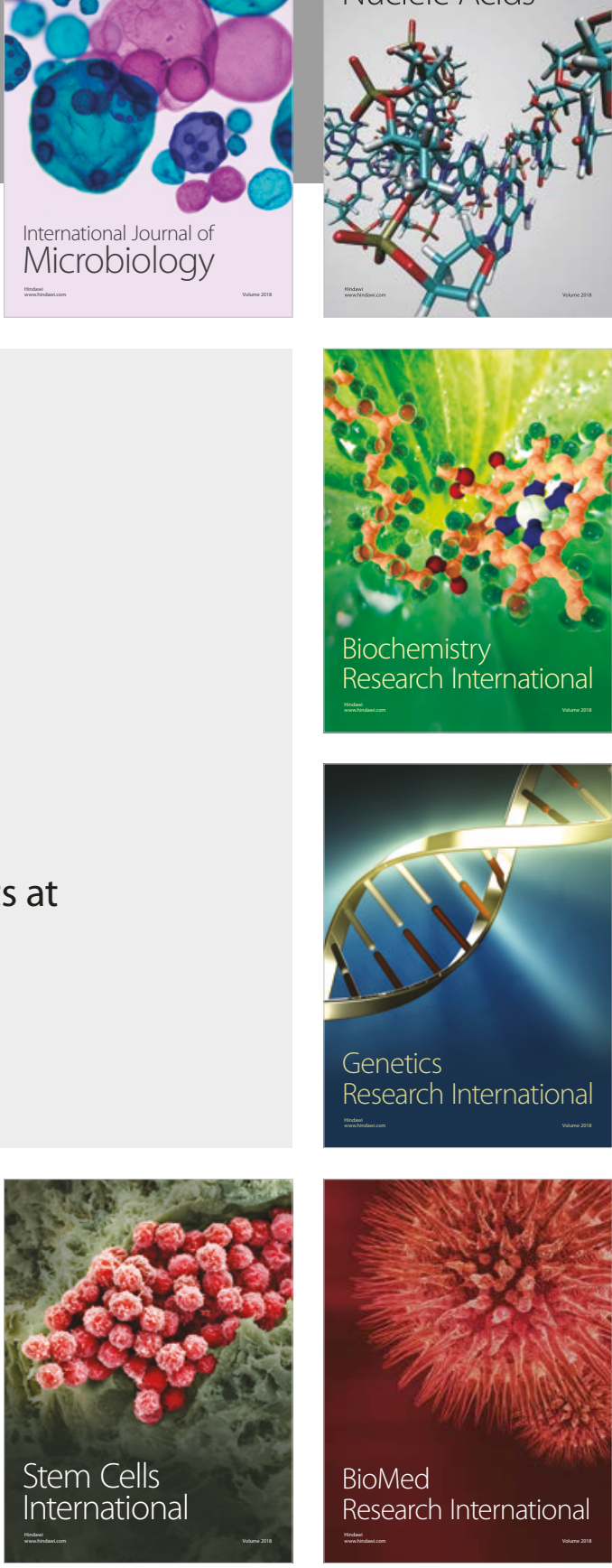
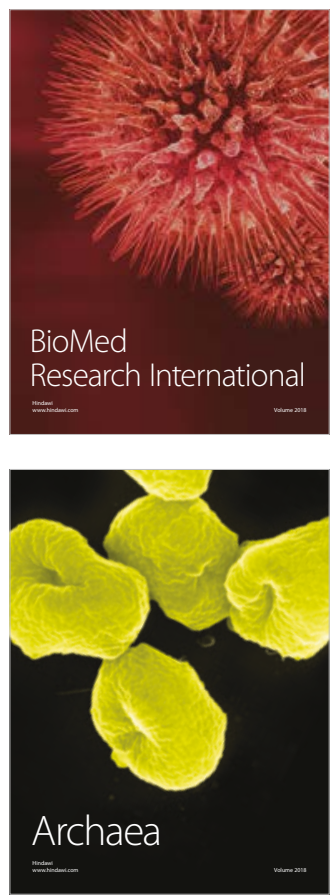